\title{
MAGIC discovery and observation of the candidate extreme BL Lac object RBS 0723
}

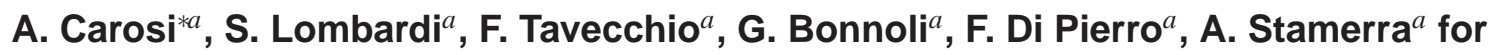 the MAGIC Collaboration}

E-mail: alessandro.carosi@oa-roma.inaf.it

${ }^{a}$ INAF, National Institute for Astrophysics, I-00136 Rome, Italy;

\begin{abstract}
The BL Lac object RBS 0723 belong to the category of "extreme" BL Lac object (EHBL). These sources are known to show an extreme frequency of their synchrotron and inverse Compton peaks in the spectral energy distribution (in the hard $\mathrm{X}$-ray and $\mathrm{TeV}$ bands respectively). Furthermore, they are characterized by the extreme hardness of the UV-X-ray and (de-absorbed) $\mathrm{TeV}$ continua. These characteristics are explained in a leptonic synchrotron+SSC emission model under the assumption that the energy distribution of the emitting relativistic electrons has a large value of the minimum Lorentz factor $\left(10^{5}\right)$ and a rather low magnetic field intensity $\left(10^{-2}\right.$ $\mathrm{G})$. This aspect points out a significant difference with respect to the parameters usually used in "classical" TeV BL Lacs modelling. Thus, EHBL are very interesting targets, that allow to investigate the properties of blazars belonging to a poorly known category of sources described by peculiar values of the relevant physical quantities of the jets. Also, the hardness of the $\mathrm{TeV}$ spectrum provides the opportunity to use these sources to probe and test different models of the extragalactic background light and intergalactic magnetic fields.
\end{abstract}

MAGIC performed an observational campaign on RBS 0723 between December 2013 and April 2014. The source was detected on January 2014 with a statistical significance of more than 5 standard deviations. The non-detection in the earlier data taken in December points to a possible VHE flux variability.

The 34th International Cosmic Ray Conference,

30 July- 6 August, 2015

The Hague, The Netherlands

\footnotetext{
* Speaker.
} 


\section{Introduction}

Blazars are a sub-class of Active Galactic Nuclei (AGN) characterized by a spectral energy distribution (SED) dominated by the relativistically-boosted non-thermal emission originating from a relativistic outflow (jet) aligned with our line-of-sight. Thanks to the presence of this jet-dominated emission, blazars are sources easily detectable up to the very high energy (VHE) accounting, in fact, the large majority of extra-galactic objects detected by Cherenkov telescopes above $\sim 100 \mathrm{GeV}$. Emission of VHE $\gamma$-ray is indeed, one of the distinctive features of blazars. The SEDs of these objects are characterized by a typical double-hump shape corresponding to two broad emission components. These SEDs have been traditionally described within the framework of a leptonic Synchrotron-self Compton (SSC) model. Within this scenario, low-energy photons (up to X-ray band) are produced by synchrotron emission while higher energy emission is explained as a product of the inverse Compton (IC) scattering of relativistic electrons on the synchrotron seed photons. However, while the synchrotron emission as explanation of the low energy hump is a rather accepted scenario, alternative models have been proposed for the high-energy component. Models based on hadronic processes have been proposed as viable scenarios where VHE photons can be originated by proton synchrotron mechanism [2] or, alternatively, by interactions initiated by ultrarelativistic hadrons [1].

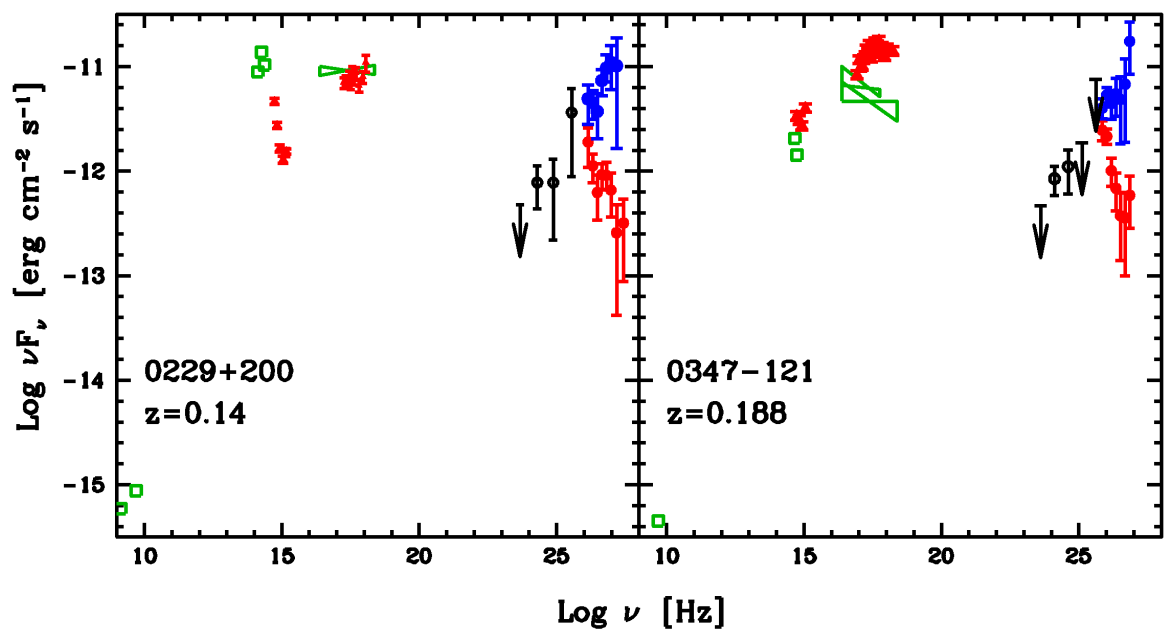

Figure 1: Spectral energy distributions of the EHBL prototypes 1ES 0229+200 (left) and1ES 0347-121 (right). Blue points are the de-absorbed TeV spectrum. Picture from [3].

For the majority of blazars, the high-energy emission peaks usually in the 1-100 GeV range, however, a small sub-sample BL Lac object shows a rather hard VHE spectrum peaking up even the $\mathrm{TeV}$ energies when de-absorbed for the extragalactic background light attenuation (EBL) [3, 4]. For such sources, an extreme hardness of spectrum is visible both in the X-ray band where the low-energy peak emission lies in the hard-X region as well as in the HE and VHE range where the spectrum is sometimes hard enough not to allow the detection of the source by Fermi-LAT. These properties have lead to the definition of extreme blazars (EHBL - Extreme High peaked BL 
Lac). The prototypes of these objects are traditionally reported to be the well-known TeV-emitters 1ES 0229+200 and 1ES 0347-121 (Fig. 1)

\section{EHBL properties}

Within the SSC modeling of blazars, the phenomenology of EHBL is usually interpreted by means of parameters rather different with respect to the standard used for normal HBL. According to [5], the unusual hard and TeV-peaked VHE spectrum (together with large X-ray/UV flux ratio) could be generated by an electron population of extremely high minimum Lorenz factor $\left(\gamma_{\mathrm{e}, \mathrm{min}} \approx\right.$ $\left.10^{5}\right)$. This value is significantly different from the averaged value conventionally used in modeling non-extreme blazars $\left(\gamma_{e, \min } \lesssim 10^{3}\right)$. This could significantly challenge particle acceleration models as described in [5]. Furthermore, EHBL are known to be a relatively steady sources over several years time scale [6]. This strongly differs from what it is observed for classical HBL that have in the strong time-variability, one of the most distinctive characteristics. The lack of variability, together with the extreme de-absorbed $\mathrm{TeV}$ spectra, has been also interpreted in a framework in which the gamma-rays are not originating in the jet but are produced by propagating UHE protons accelerated in the jet and beamed toward the Earth.

The peculiarly hard $\gamma$-ray spectrum of EHBL makes them particularly suitable to probe extragalactic background light (EBL) or the extragalactic magnetic field (EGMF). Attempts to derive the intensity of the EGMF using the observed VHE spectrum an the upper limits derived by LAT for the $\mathrm{GeV}$ band provides values in the range $10^{-18}-10^{-15} \mathrm{G}$ [7].

\subsection{RBS 0723}

$\operatorname{RBS} 0723\left(\mathrm{RA}(\mathrm{J} 2000)=08^{\mathrm{h}} 47^{\mathrm{m}} 12.9^{\mathrm{s}}, \operatorname{Dec}(\mathrm{J} 2000)=+11^{\circ} 33^{\prime} 50^{\prime \prime}\right)$ is a BL Lac object at $z=0.198$ identified as a bright X-ray source in the ROSAT All Sky Survey. It has been also observed by Swift, revealing a bright and hard X-ray continuum. RBS 0723 belongs to the 1FHL catalogue [8], showing a hard $\Gamma_{1 \mathrm{FHL}}=1.4 \pm 0.4$ and bright $\left(\mathrm{F}>10 \mathrm{GeV}=9.6 \times 10^{-11} \mathrm{ph} \mathrm{cm}^{-2} \mathrm{~s}^{-1}\right)$ emission above $10 \mathrm{GeV}$. Moreover, it is also detected by LAT above $30 \mathrm{GeV}$ with a significance $>6 \sigma[8]$.

\section{MAGIC Observations and data analysis}

First MAGIC observations were carried out between 2013 December and 2014 January during dark time and at low zenith angle $\left(<35^{\circ}\right)$. After the first detection of January [9], observation on RBS 0723 were carried on until April 2014 and, later on, for few more hours between October and November 2014. The overall data sample consists of about $52 \mathrm{~h}$ of data. After data quality cuts mainly based on the stereo trigger rate stability, the cleaned data sample used for the results presented in this contribution, amounts to $45 \mathrm{~h}$ of data in the zenith angle range between $15^{\circ}$ and $35^{\circ}$ taken with the MAGIC standard stereo configuration. The observations were carried out in the so-called wobble mode, in which the pointing direction alternates every $20 \mathrm{~min}$ between four positions, offset by $\pm 0.4^{\circ}$ from the source. The achieved energy threshold is $\sim 80 \mathrm{GeV}$ after analysis cuts. 
The data analysis was performed using the standard MAGIC analysis package MARS [10]. After the calibration of the single events recorded by each telescope, the data were processed with a stanadard cleaning process as described in [12] . For the gamma/hadron separation and gamma-direction estimation, a multivariate method based on a random forest (RF) algorithm was applied [13]. This algorithm employs some Cherenkov image parameters [14] to compute a gamma/hadron discriminator called hadronness by comparison with Monte Carlo gamma-ray simulations. The energy of the events was estimated by averaging individual energy estimators for both telescopes based on look-up tables. The detection of the possible gamma-ray signal is achieved through the so-called $\theta^{2}$ plot, i.e., the comparison between the distributions of the square angular distance between the reconstructed position of the source and its nominal position in the signal and background regions for energies above the threshold. The significance of the signal is evaluated using single cuts in hadronness and $\theta^{2}$ and according to Eq. 17 of [15].

\section{Results}

Fig. 2 show the achieved $\theta^{2}$ plot (left panel) and the spatial distribution of the RBS 0723 significance (sky plot, right panel) obtained from the full data sample.
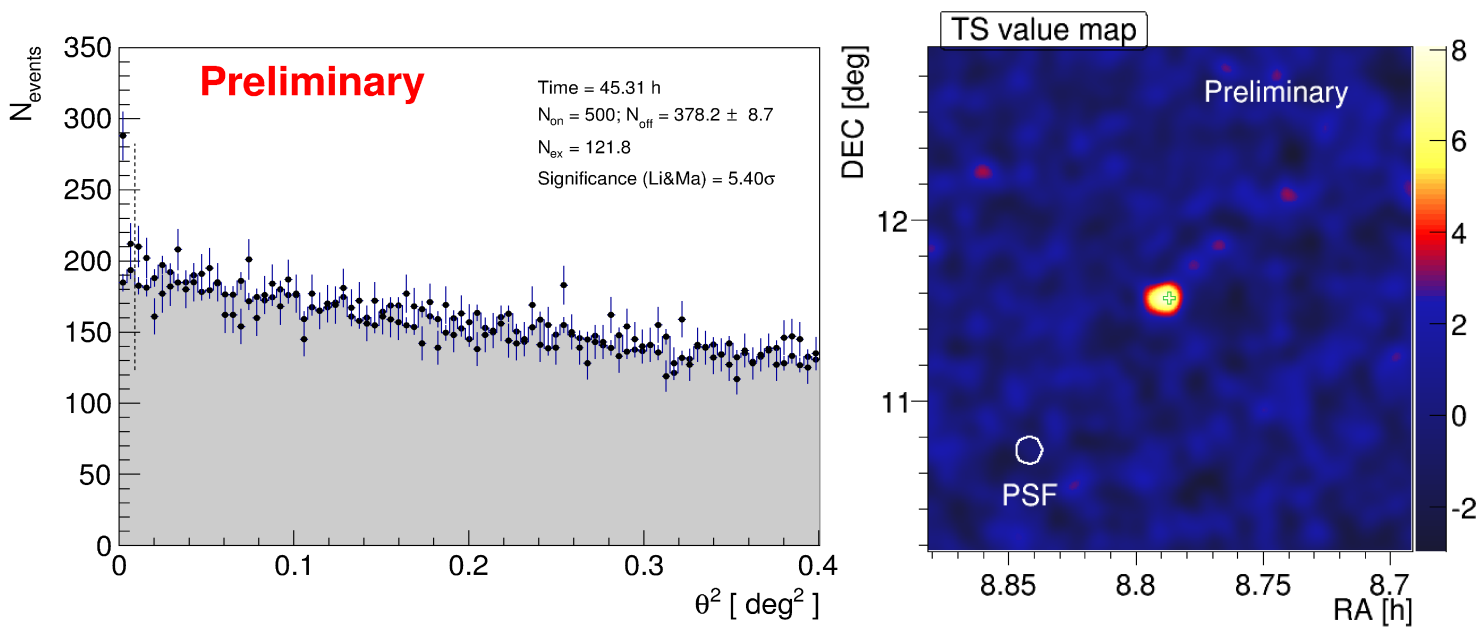

Figure 2: Left:Distribution of the $\theta^{2}$ parameter for RBS 0723 signal (black points) and background (gray area) regions for the full data sample above $\sim 200 \mathrm{GeV}$. The region between zero and the vertical dashed line represents the signal region. Right: spatial distribution of the significance of the excesses events from the sky region of RBS 0723. The color palette reports the test statistic value (TS), which is defined as the significance from Eq. 17 in [15] applied on a smoothed and modeled background estimation.

We found around $\sim 122$ excess events within the signal region corresponding to a significance of $5.4 \sigma$ according to [15]. It has to be remarked that the source is clearly detected when using analysis cuts optimized for energies above $\sim 200 \mathrm{GeV}$ while it is not detected in the MAGIC low energy band close to the energy threshold as it is to be expected for an extreme source.

Although the first data collected during 2013 do not show significant hint of signal, the source was clearly detected above $200 \mathrm{GeV}$ with a statistical significance of more then 5 standard de- 
viations on January 2014 when the VHE emission measured with MAGIC had an integral flux of $4.4 \times 10^{-12} \mathrm{~cm}^{-2} \mathrm{~s}^{-1}$, corresponding to about $2 \%$ of the Crab nebula (CU) flux above $200 \mathrm{GeV}$ [9]. Optical observations performed with the KVA telescopes also revealed an increasing flux in the R-band in January 2014. Subsequent observations during the following two months marginally provide significant detection. Fig. 3 shows the monthly-binned light curve obtained by MAGIC at energy above $200 \mathrm{GeV}$ for the complete observation period. A steady emission is excluded by our data at $\sim 2 \sigma$ confidence level.

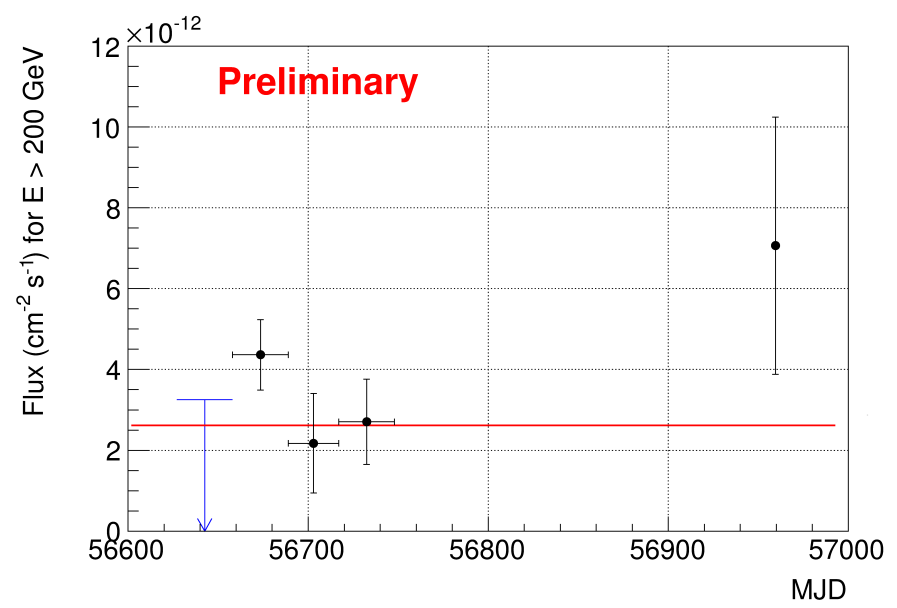

Figure 3: MAGIC monthly light curve of RBS 0723 above $200 \mathrm{GeV}$. The red line at $2.6 \times 10^{-12} \mathrm{~cm}^{-2} \mathrm{~s}^{-1}$ $(\sim 1.2 \% \mathrm{CU})$ represents the data points fit assuming a steady emission.

The shape of the synchrotron component of RBS 0723 resembles that typically shown by the EHBL and can be reproduced assuming a relatively large value of $\gamma_{\min }$. In turn, this also imply a hard SSC component, in agreement with the LAT data. However, contrary to the EHBL, the VHE spectrum of RBS 0723 seems to be soft, although the uncertainty is so large that we cannot make a strong conclusion.

\section{Conclusion}

MAGIC observed the candidate extreme BL Lac object RBS 0723 between December 2013 and November 2014. The source was clearly detected on January 2014 with an integral flux of about $2 \%$ of Crab nebula flux above $200 \mathrm{GeV}$. The marginally detection in subsequent months suggests a possible VHE flux variability that is quite exceptional on its own as EHBL are thought to be relatively steady source. Furthermore, our preliminary spectral analysis reveal a relatively soft VHE spectrum although the large uncertainties in the measured spectral index do not allow, for the time being, to draw firmer conclusion.

Detailed spectral information will be the target of a forthcoming dedicated publication.

\section{Acknowledgments}

We would like to thank the Instituto de Astrofisica de Canarias for the excellent working conditions at the Observatorio del Roque de los Muchachos in La Palma. The financial support of the German BMBF 
and MPG, the Italian INFN and INAF, the Swiss National Fund SNF, the ERDF under the Spanish MINECO (FPA2012-39502) and MECD (FPU13/00618), and the Japanese JSPS and MEXT is gratefully acknowledged. This work was also supported by the Centro de Excelencia Severo Ochoa SEV-2012-0234, CPAN CSD2007-00042, and MultiDark CSD2009-00064 projects of the Spanish Consolider-Ingenio 2010 programme, by grant 268740 of the Academy of Finland, by the Croatian Science Foundation (HrZZ) Project 09/176 and the University of Rijeka Project 13.12.1.3.02, by the DFG Collaborative Research Centers SFB823/C4 and SFB876/C3, and by the Polish MNiSzW grant 745/N-HESS-MAGIC/2010/0.

\section{References}

[1] Bottcher, M.,Reimer, A., Sweeney, K. et al. Leptonic and Hadronic Modeling of Fermi-detected Blazars, ApJ 768 (2013) 54

[2] Aharonian, F.A. TeV gamma rays from BL Lac objects due to synchrotron radiation of extremely high energy protons., New Astronomy 5 (2000) 377

[3] Bonnoli, G., Tavecchio, F., Ghisellini, G., et al. An emerging population of BL Lac with extreme properties: towards a class of EBL and cosmic magnetic field probes?, MNRAS 451 (2015) 5130

[4] Tavecchio, F., Ghisellini, G., Bonnoli, G. et al. Extreme TeV blazars and the intergalactic magnetic field, MNRAS 414 (2011) 3566

[5] Tavecchio, F., Ghisellini, G., Ghirlanda, G. et al. The hard TeV spectrum of 1ES 0229+200: new clues from Swift, MNRAS 399 (2009) 59

[6] Aliu, E., Archambault, S., Arlen, T. et al (VERITAS Collaboration) A Three-Year Multi-Wavelength Study of the Very High Energy Gamma-ray Blazar 1ES 0229+200, ApJ 782 (2014) 13

[7] Vovk, I., Taylor, A. M., Semikoz, D et al. Fermi/LAT observations of 1ES 0229+200: implications for extragalactic magnetic fields and background light, ApJ Letters 747 (2012) L14

[8] Ackermann, M., Ajello, M., Allafort, A. et al. The first Fermi-LAT catalog of sources above $10 \mathrm{GeV}$, ApJS 209 (2013) 34

[9] Mirzoyan, R. for the MAGIC Collaboration Discovery of very high energy gamma-rayemission from RBS 0723 with the MAGIC telescopes, ATel 5768

[10] R. Zanin et al., MARS, the MAGIC Analysis and Reconstruction Software, In Proc. of the 33st International Cosmic Ray Conference, Rio de Janeiro, Brasil (2013)

[11] Albert, J., Aliu, E., Anderhub, H., et al.,FADC signal reconstruction for the MAGIC telescope, Nucl. Instrum. Meth., A59 4 (2008) 407

[12] Aleksić, J. et al. (MAGIC collaboration), The major upgrade of the MAGIC telescopes, Accepted for publication in Astropar ticle Physics, arXiv:1409.5594v3

[13] Albert, J., Aliu, E., Anderhub, H., et al., Implementation of the Random Forest Method for the Imaging Atmospheric Cherenkov Telesco pe MAGIC, Nucl. Instrum. Meth., A588 (2008) 424

[14] Hillas, A. M. 1985, Cherenkov light images of EAS produced by primary gamma, In Proc. of 19th , International Cosmic Ray Conference, La Jolla, (1985)

[15] Li, T.-P., \& Ma, Y.-Q., Analysis methods for results in gamma-ray astronomy, ApJ 272 (1983) 317 